\title{
Optics of Ionization Cooling Channels Under the Influence of Space Charge
}

\author{
Ben Loseth ${ }^{1}$, Martin Berz ${ }^{1}$, He Zhang ${ }^{2}$, Pavel Snopok ${ }^{3}$ and Josiah Kunz ${ }^{3}$ \\ 1. Department of Physics and Astronomy, Michigan State University, East Lansing, Michigan, USA \\ 2. Center for Advanced Studies of Accelerators, Jefferson Laboratory, Newport News, Virginia, USA \\ 3. Department of Physics, Illinois Institute of Technology, Chicago, Illinois, USA
}

Lepton colliders have a significant advantage over their hadron counterparts in that hadron collisions are inefficient and complicated by secondary quark interactions. A muon collider could be used for high energy studies of lepton collisions without the limitations on energy due to synchrotron radiation. The muon beam is produced by sending protons through a target, producing pions which in turn decay into muons with a large momentum spread. For a muon collider, the six-dimensional (6D) phase space volume of the muon beam must be reduced to accelerate it further for injection into a storage ring. Ionization cooling is currently the only feasible method for cooling the beam within a muon lifetime of $2.2 \mu$ s. One key technical challenge for a muon collider is the demonstration of the process of ionization cooling. In order for a full 6D ionization cooling experiment to be constructed, a baseline lattice design has to be studied and selected based on detailed simulations [1].

To reduce the transverse emittance, the beam is strongly focused with high magnetic fields and subsequently sent through an absorber material to reduce overall momentum. The beam regains longitudinal momentum in RF cavities which results in an overall loss in transverse emittance. The behavior of the transverse emittance is described by:

$$
\frac{d \varepsilon_{n}}{d s}=-\frac{1}{\beta^{2}} \frac{d E_{\mu}}{d s} \frac{\varepsilon_{n}}{E_{\mu}}+\frac{1}{\beta^{3}} \frac{\beta_{T} E_{s}^{2}}{2 E_{\mu} m_{\mu} c^{2} L_{R}}, \quad \varepsilon_{n, \min }=\frac{\beta_{T} E_{s}^{2}}{2 \beta m_{\mu} c^{2} L_{R}\left|\frac{d E_{\mu}}{d s}\right|},
$$

where $\varepsilon_{n}$ is the normalized transverse emittance, $c$ is the speed of light, and $\beta=v / c, E_{\mu}, m_{\mu}$ are the muon velocity, energy, and mass. The transverse betatron function within the absorber is $\beta_{T}, d E_{\mu} / d s$ is the energy loss per unit length in the absorber, $L_{R}$ is the radiation length of the absorber material, and $E_{S}$ is the characteristic scattering energy. The left equation details the effects of cooling (first term) and heating (second term), and when the cooling rate equals the heating rate, we can find the minimum normalized transverse (or equilibrium) emittance $\left(\varepsilon_{n, \min }\right)$ for a given absorber material and focusing field.

To maximize cooling, materials with low- $\mathrm{Z}$ and large radiation length (such as $\mathrm{LH}_{2}, \mathrm{Li}, \mathrm{Be}$ ) are used in order to minimize multiple scattering. A high gradient RF field and high absorber density, the combination of which determines the energy loss per unit length $d E_{\mu} / d s$, are used along with solenoids arranged such that there is strong focusing at absorbers, namely having small $\beta_{T}$. Cooling typically occurs around a muon momentum of $200 \mathrm{MeV} / \mathrm{c}$, where the curvature of the Bethe-Bloch formula (to determine absorber $\left.d E_{\mu} / d s\right)$ is favorable. The cooling channel is tapered into stages in order to vary the solenoid focusing, RF frequency and gradient, and geometry such that the minimum normalized emittance decreases with the beam [2-4].

The longitudinal momentum spread is reduced using the concept of emittance exchange. This process involves applying a magnetic field to create dispersion in the beam at wedge shaped absorbers, such that particles with higher energy pass through more absorber than those with lower energy, resulting in 

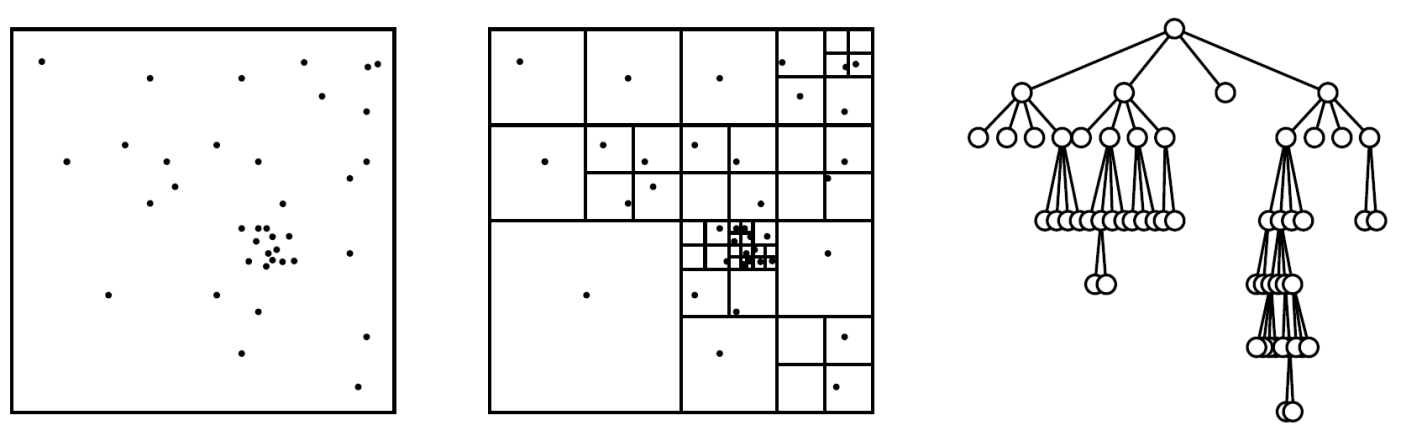

Figure 1. An example of the decomposition of a two dimensional charge distribution and resulting hierarchical structure for the FMM.

an overall reduction in the energy spread of the beam. The cooling process is essentially a transfer of emittance from the longitudinal to transverse direction combined with transverse ionization cooling for emittance reduction in all six dimensions.

There are several challenges to resolve in order to demonstrate the feasibility of an ionization cooling channel. As the size of the beam is reduced, Coulomb repulsion in the beam restricts emittance reduction. Accurate calculation of this space charge effect is necessary. In addition, time-dependent absorber degradation is a factor to consider, as the minimum normalized emittance is sensitive to changes in absorber properties such as $d E / d s$. Also, late stages of the cooling channel have high field solenoids that negatively affect RF performance.

To investigate the effect of space charge, a method has been implemented in COSY INFINITY [5] to achieve efficient and accurate calculation of the interparticle Coulomb forces based on variants of the Fast Multipole Method (FMM). This method divides an arbitrary charge distribution into small boxes with a hierarchical structure as shown in Figure 1. It then computes the multipole expansions and local expansions of charges far from the observer to achieve a computational efficiency that scales with the number of particles, $N$, and computational errors scaling with a high power of the expansion order. The FMM algorithm is especially suited for beam dynamics simulations because of the efficiency and low computational error compared to other space charge algorithms.

Other methods, such as the particle-particle interaction (PPI) and the particle in cell (PIC), while computationally efficient, incur excess error due to modeling and statistics. The PPI method uses macroparticles and assumes a particular distribution. The PIC method places the charge distribution onto a mesh, solves the Poisson equation on mesh points and interpolates between mesh points to find the field on each particle. Both of these methods suffer from an inability to precisely handle complicated charge distributions. This difficulty is overcome in the FMM by decomposing the charge distribution into boxes according to the charge density such that there are a pre-specified number of particles in each box to efficiently and accurately compute the multipole expansions [6,7].

In COSY, we use an 8th order Runge-Kutta integrator with automatic step size control. This integrator automatically selects a time step size based on a prespecified error bound. This allows selection of large initial timesteps, as the integrator reduces the stepping at difficult areas in the cooling cell which typically occur at the boundaries of elements. However, tracking particles through elements with the boundaries behaving as step functions is unnecessarily difficult for the integrator, resulting in very small timestepping 

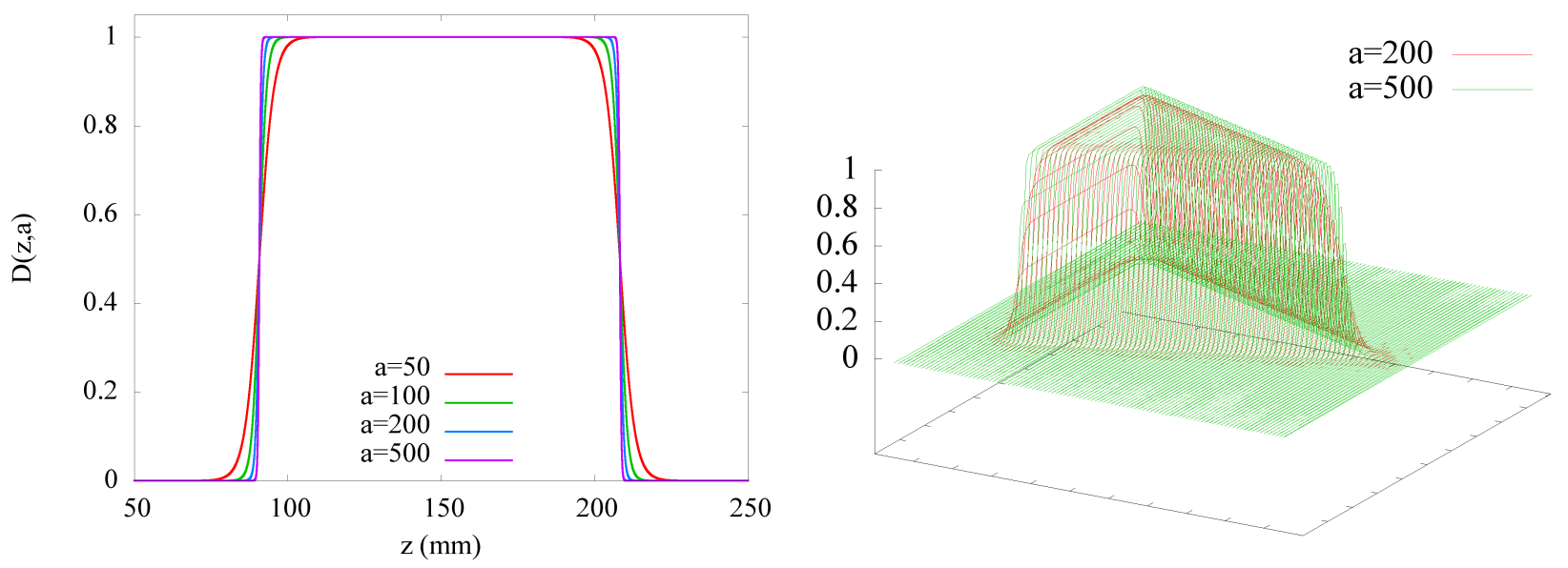

Figure 2. Rectangular and wedge-shaped beam elements using Eq. (1) (left) and Eq. (2) (right) for various Enge function falloffs.
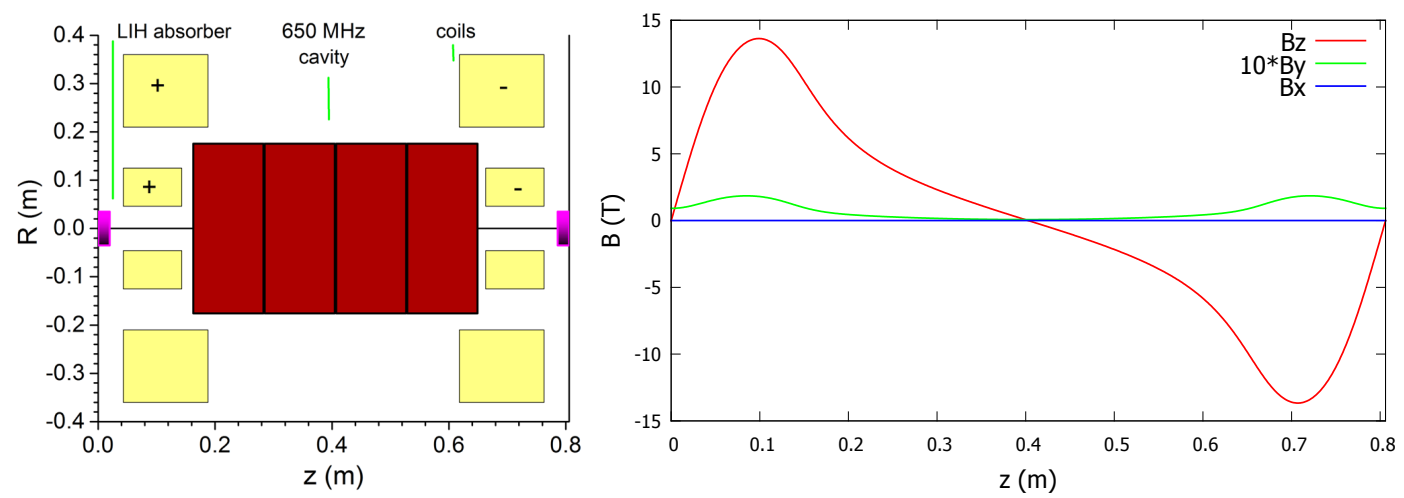

Figure 3. Diagram (left) and magnetic field in the $x, y$, and $z$ directions (right) of a late-stage cooling cell.

at boundaries in order to reach the desired error bound. To improve the efficiency of the integrator, it is advantageous to continuously vary the density of the elements. This is accomplished by applying functions of the form:

$$
D(z, a)=\frac{1}{1+\exp \left(a\left(z_{s}-z\right)\right)} \frac{1}{1+\exp \left(a\left(z-z_{e}\right)\right)},(1) \quad \text { or } \quad D(\vec{r}, a)=\Pi_{i=1}^{n} \frac{1}{1+\exp \left(a\left(\left|\vec{r}-\vec{r}_{i}\right|-R\right)\right)}
$$

For a rectangular element with start and end positions $z_{s}$ and $z_{e}$, Eq. (1) has the property of being zero for positions outside and unity for positions inside the element while varying smoothly at the boundary. More complicated objects can be described by a product of immense overlapping spheres as in Eq. (2). To do so, we find a centerpoint $\left(r_{i}\right)$ for a sphere of large radius $\left(R=10^{3} \mathrm{~m}\right)$ such that the surface of the sphere will coincide with one of the elements' faces. For a large enough radius, the curvature of the sphere becomes negligible compared to the size of the beam element, and the sharpness of the element falloff is controlled by the constant $a$. Figure 2 shows examples of these continuous density functions [8]. We have performed simulations of the late stages of a proposed cooling channel design in both COSY [5] and G4Beamline [9], with and without space charge effects. G4Beamline is one of the de-facto codes for muon beam analysis and provides a PIC space charge calculation algorithm. Stochastic effects and particle decays have yet to be implemented in COSY (see also [10]), so this functionality has been disabled to ensure a clear comparison between the codes. 

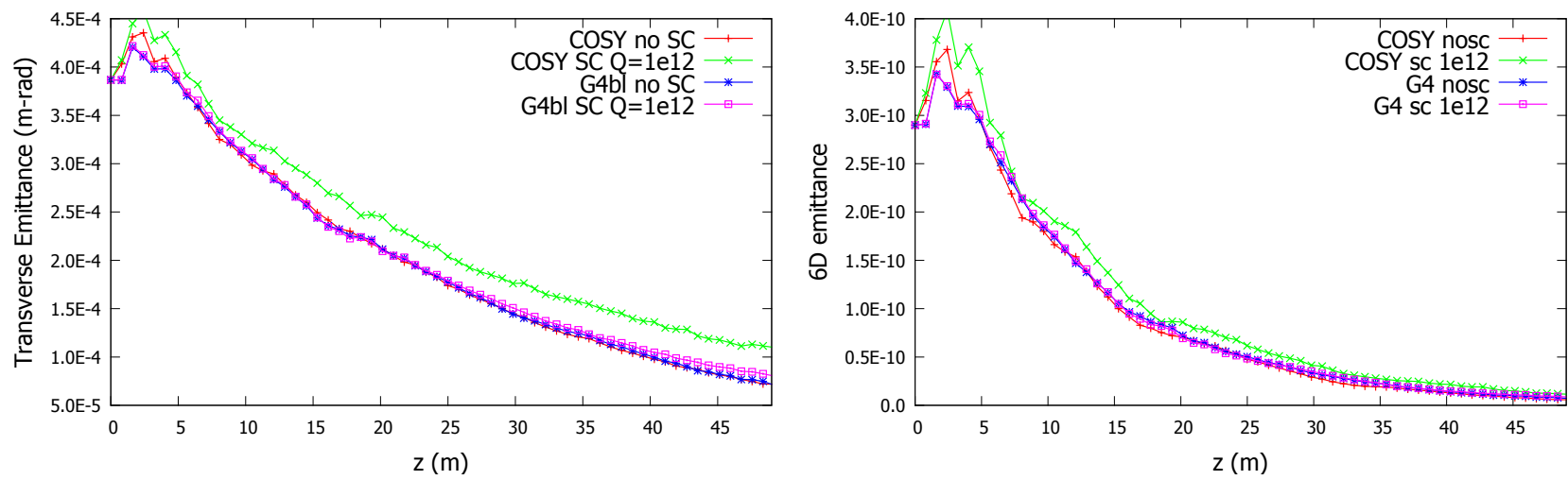

Figure 4. Transverse emittance (left) and 6D emittance (right) behavior with and without space charge.

One cell of this late-stage cooling channel consists of four $26 \mathrm{MV} / \mathrm{m}, 650 \mathrm{MHz}$ RF pillboxes, two 120 degree Lithium Hydride $(\mathrm{LiH})$ wedge absorbers, and a solenoid arrangement producing a maximum on-axis field of $12.9 \mathrm{~T}$. The solenoids have opposing currents and a small tilt to create dispersion in the $x$ direction at the absorbers. Figure 3 depicts the cell geometry and magnetic field. The simulations were run using a bunch of $10^{4}$ muons and Figure 4 depicts the transverse and 6D emittance over approximately 60 cells. For the purposes of space charge, the bunch was given a total charge of $10^{12}$. The $6 \mathrm{D}$ emittance is reduced by an order of magnitude over this stage of cooling, showing promising emittance reduction if combined with previous stages. COSY predicts a larger space charge effect in the transverse direction, likely due to a difference in the space charge algorithms. Despite this difference, the behavior of the emittance agrees well between the two codes.

In this article, we explained the specifics of muon beam cooling, discussed the methods of cooling channel simulations, and presented results of the simulation. Our future plans involve incorporating an entire cooling channel in COSY while investigating the differences in longitudinal emittance behavior, and further optimizing the efficiency of COSY simulations [11].

\section{References:}

[1] Muon Accelerator Program; http://map.fnal.gov.

[2] D Neuffer, Nuclear Instruments and Methods A 532 (2004), p. 26.

[3] R. Palmer et al, Physical Review Special Topics - Accelerators and Beams 8 (2005), p. 061003.

[4] D Stratakis et al, Physical Review Special Topics - Accelerators and Beams 16 (2013), p. 091001.

[5] M Berz and K Makino, "COSY INFINITY version 9.1 programmer's manual”, MSUHEP-101214, (Michigan State University, East Lansing, 2011), http://cosyinfinity.org.

[6] H Zhang, "The fast multipole method in the differential algebra framework for the calculation of 3D space charge fields", Ph.D thesis, (Michigan State University, East Lansing, 2013).

[7] H Zhang and M Berz, Nuclear Instruments and Methods in Physics Research A 645 (2011), p. 338344.

[8] M Berz, "Modern map methods in particle beam physics", (Academic Press, San Diego, 1999).

[9] T Roberts, "G4Beamline”, (2013), http://www.muonsinternal.com/muons3/G4beamline.

[10] J Kunz et al, in these proceedings.

[11] We are grateful for the financial support provided by the URA Visiting Scholars at Fermilab Program under award \#13-S-42, and US Department of Energy under grant DE-FG02-08ER41546. We thank K Makino for excellent and useful comments. 